\title{
Development of Measurement Device of Track Irregularity using Inertial Mid-chord Offset Method
}

\author{
Eiji YAZAWA \\ Assistant Senior Researcher, \\ Track Geometry \& Maintenance, Track Technology Div. \\ Kunio TAKESHITA \\ Chief Engineer, \\ Opto-Precision Systems Div., Shonan Business G., Hitachi Electronics Engineering Co., Ltd
}

An inevitable problem of the inertial measuring method is that the waveform is distorted by the high-pass filtering process for stabilizing the double integration of acceleration. We designed a method to overcome this problem, by combining characteristics of the inertial measuring method and mid-chord offset method. We call it an "inertial midchord offset method." By using a prototype track inspection device that applies the new method, we confirmed that it provides track irregularity waveforms similar to those obtained by the mid-chord offset method. Moreover, as part of that equipment, we developed a sensor to measure vertical and horizontal rail displacements at the same time.

Keywords : track inspection, inertial measuring method, mid-chord offset method, inertial mid-chord offset method, two-axis rail displacement sensor

\section{Introduction}

The mid-chord offset method, which is currently the mainstream methods to measure vertical and horizontal track displacements by track inspection cars, uses three measuring axes arranged in line. The center axis measures the offset of the rail from the chord that connects the two end axes. The system to set a measuring axis at the center makes the car body and running gears complicated and increases the cost of inspection car. The asymmetrical chord offset method will reduce the restriction of car design. As this method still uses three measuring axes, however, it will not reduce the cost of inspection car.

On the other hand, the inertial measuring method, which is based on the principle that the double integration of acceleration gives displacement, uses only one measuring axis. Therefore, the design of inspection car is flexible and the measuring system can be mounted on a commercial railway vehicle. Inspection cars of this type will be manufactured at low costs. However, this method requires high-pass filtering process of measurements to stabilize the double integration, which would otherwise distort measured track waveforms.

For this purpose, we combine the characteristics of the inertial and mid-chord offset methods to obtain distortion-free mid-chord offset irregularity waveforms, which we call an "inertial mid-chord offset method." This paper introduces the theory of the new method, test results obtained using the method for an existing track inspection car, and an outline of prototype measuring device to apply the method.

\section{Theory of inertial mid-chord offset method}

Figure 1 shows the basic composition of the inertial mid-chord offset method, which does not differ much from that of the conventional inertial method, except the characteristic "integration and mid-chord offset composite calculation" by a waveform processing unit ${ }^{1 \text {. }}$.

Figure 2 shows the principle of integration and midchord offset composite calculation, in which Fig. 2(a) shows the frequency characteristic function of the double integration of acceleration. The gain of this function is infinite when the frequency is 0 , which makes the double integration unstable. Figure 2 (b) shows the inspection characteristic function of the mid-chord offset method for

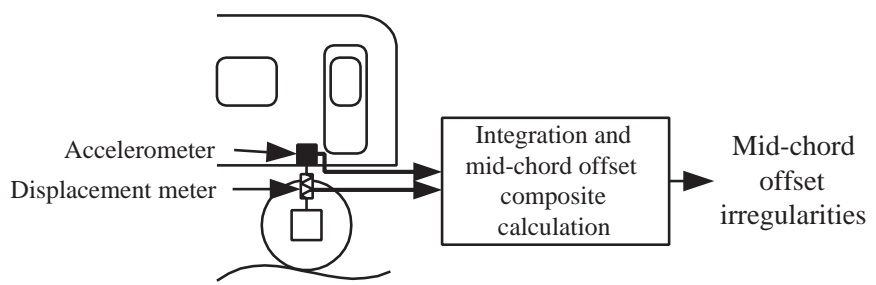

Fig. 1 Basic composition of the inertial mid-chord offset method

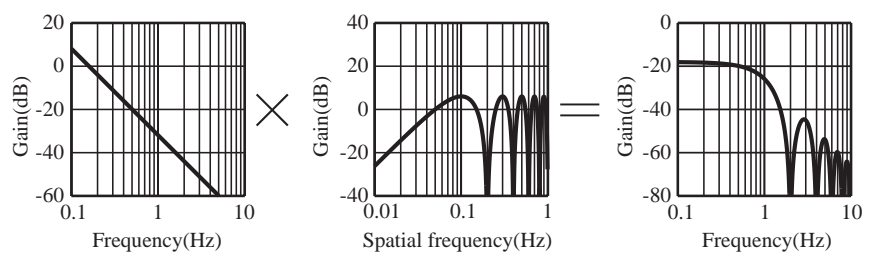

Fig. 2 Composition of double integration and mid-chord offset characteristics (integration and mid-chord offset composite calculation) 
the chord length of $10 \mathrm{~m}$ as an example. The function to express the inspection characteristic of the mid-chord offset method attenuates in the low frequency range at the rate equal to the increasing rate of the double integration in the range. If acceleration is processed by the function in Fig. 2 (c) that compounds these two functions, therefore, the gain takes a finite value even when the frequency is 0 . This ensures stable calculation and outputs mid-chord offset waveforms that are normally used for track inspection.

The composite characteristic function in Fig. 2 (c) is expressed by the following equation.

$G_{2}(s) \cdot G_{1}(s)=\frac{1}{s^{2}}\left[\exp \left(-\frac{s}{2 \omega_{1}}\right)-\frac{1}{2}\left\{1-\exp \left(-\frac{s}{\omega_{1}}\right)\right\}\right] \cdots \cdots \cdots \cdots \cdots(1)$ $\omega_{1}=\frac{v}{l_{1}}$

where,

$s$ : Laplace operator

$G_{1}(s)$ : Transfer function of the mid-chord offset method

$G_{2}(s)$ : Transfer function of double integration

$v \quad$ : Running speed $(\mathrm{m} / \mathrm{s})$

$l_{1}$ : Chord length used by the mid-chord offset method

This characteristic function can be obtained theoretically by a single digital filter. The input acceleration normally, however, has large power in the high frequency range, so that filtering after direct $\mathrm{A} / \mathrm{D}$ conversion will cause errors due to aliasing. To solve this problem, we split the characteristic function into several segments for calculation.

There are several methods to split the characteristic function. One is to separate the acceleration signal into high- and low-frequency components for separate filtering and compound the results to obtain the characteristic function shown by the equation (1) ${ }^{2}$. As a more practical method, this paper introduces a method to pass the acceleration signal through an analog integrator to attenuate the high-frequency component and perform digital filtering to obtain the characteristic function in equation (1). Figure 3 shows a block diagram of this method. In the figure, $G_{20}(s)$ is a low-pass filter type integrating circuit; $G_{30}(s)$ indicates digital filter processing; and $G_{40}(s)$ is a high-pass filter.

$$
G_{6}(s)=\frac{s^{2}}{s^{2}+2 \cdot \zeta_{n} \cdot \omega_{n} \cdot s+\omega_{n}^{2}}
$$

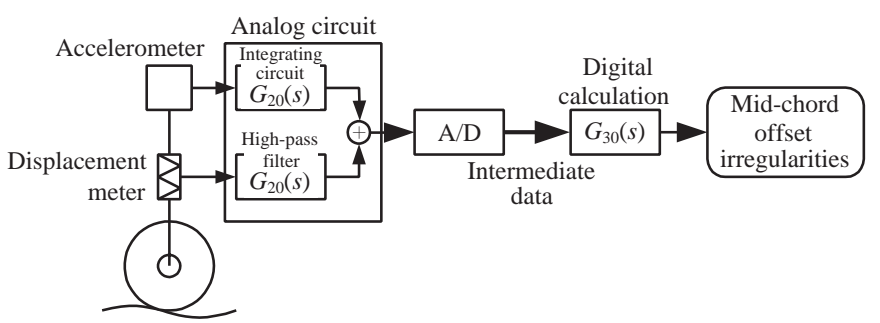

Fig. 3 Block diagram of the basic composition of the inertial mid-chord offset method

$$
\begin{aligned}
G_{20}(s) & =K_{20} \cdot G_{2}(s) \cdot G_{6}(s)=K_{20} \cdot \frac{1}{s^{2}+2 \cdot \zeta_{n} \cdot \omega_{n} \cdot s+\omega_{n}^{2}} \\
G_{30}(s) & =\frac{G_{1}(s)}{G_{6}(s)} \\
G_{40}(s) & =K_{40} \cdot \frac{s^{2}}{s^{2}+2 \cdot \zeta_{n} \cdot \omega_{n} \cdot s+\omega_{n}^{2}} \\
K_{20}(s) & =\frac{K_{3}}{K_{1}} \\
K_{40}(s) & =\frac{K_{3}}{K_{2}} \\
\omega_{n}= & \frac{v}{l_{2}} \\
& \\
\text { where, } & \\
& K_{1}: \text { Gain of accelerometer }(\mathrm{V} / \mathrm{m}) \\
& K_{2}: \text { Gain of displacement sensor }(\mathrm{V} / \mathrm{m}) \\
& K_{3}: \text { Output scale factor of displacement sensor }(\mathrm{V} / \mathrm{m}) \\
& l_{2}: \text { Acceleration smoothing length }(\mathrm{m}) \\
& \zeta: \text { Attenuation constant of analog filter } \\
& G_{6}(s) \text { Transfer function of high-pass filter } \\
&
\end{aligned}
$$

The digital filter processing to attain $G_{30}(s)$ is expressed by the following equation.

$D_{\text {out }}=\sum_{j=0}^{n} W_{31}(j) \cdot D_{\text {in }}(i-j)$

where,

$$
\left.\begin{array}{l}
W_{31}(j)=-\frac{1}{2}-\frac{1}{2} \zeta_{n} \frac{l_{1}}{n \cdot l_{2}}-\frac{1}{16}\left(\frac{l_{1}}{n \cdot l_{2}}\right)^{2} \quad(j=0) \\
W_{31}(j)=-\zeta_{n} \frac{l_{1}}{n \cdot l_{2}}-\frac{j}{2}\left(\frac{l_{1}}{n \cdot l_{2}}\right)^{2} \quad\left(0<j<\frac{n}{2}\right) \\
W_{31}(j)=1-\left(\frac{n}{4}-\frac{1}{8}\right) \cdot\left(\frac{l_{1}}{n \cdot l_{2}}\right)^{2} \quad\left(j=\frac{n}{2}\right) \\
W_{31}(j)=\zeta_{n} \frac{l_{1}}{n \cdot l_{2}}-\frac{n-j}{2}\left(\frac{l_{1}}{n \cdot l_{2}}\right)^{2}\left(\frac{n}{2}<j<n\right) \\
W_{31}(j)=-\frac{1}{2}+\frac{1}{2} \zeta_{n} \frac{l_{1}}{n \cdot l_{2}}-\frac{1}{16}\left(\frac{l_{1}}{n \cdot l_{2}}\right)^{2}(j=n)
\end{array}\right\}
$$

$n=l_{1} / \Delta l$

$n$ : Number of the segments used by the mid-chord offset method

$\Delta l$ : Sampling interval (m)

$D_{\text {in }}(i)$ : Input

$D_{\text {out }}(i)$ : Output

Figure 4 shows the spatial frequency characteristic function of this processing method when the running speed is constant.

As the characteristic function of the integration processing section is of the analog low-pass filter type as mentioned above, this method suppresses errors in A/D conversion and performs digital filtering at high preci- 


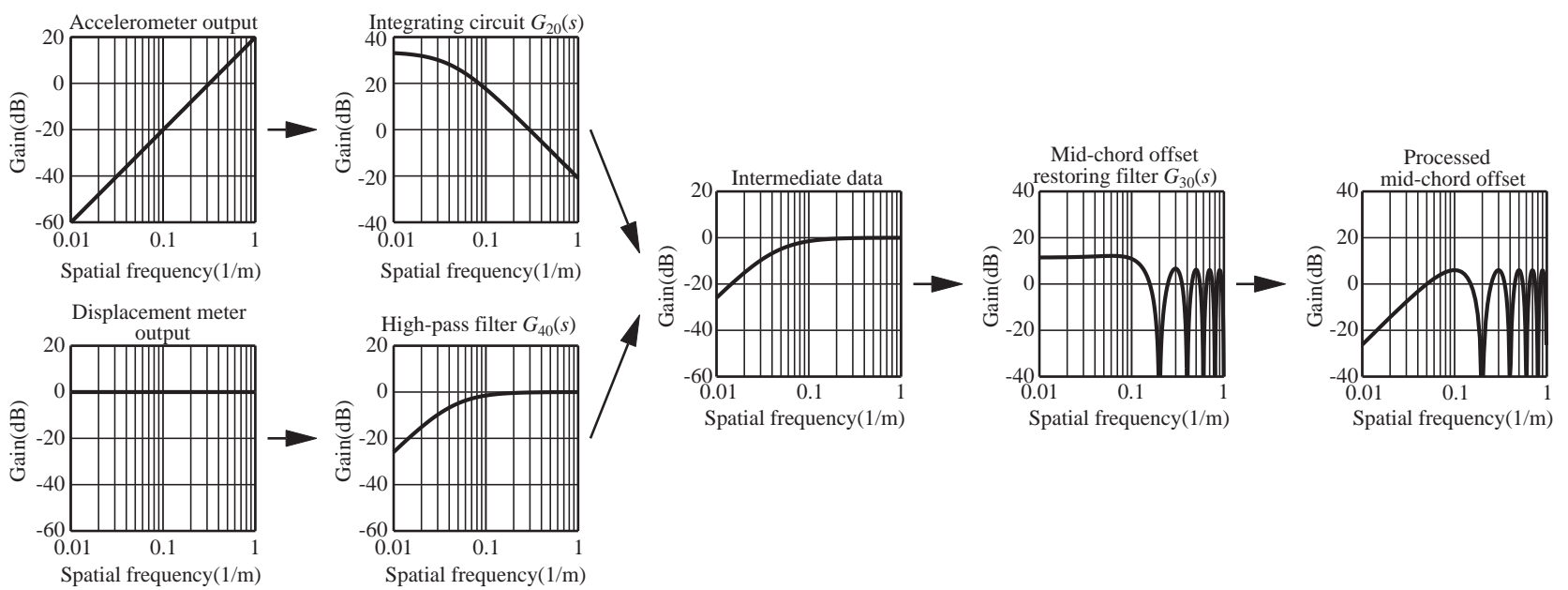

Fig. 4 Spatial frequency characteristic function of waveform processing by the inertial mid-chord offset method

sion in the restoring process of the mid-chord offset method. This digital filter also has a characteristic to compensate the phase delay occurred in the analog circuit. In this composition, the integration characteristic can be adjusted against the changes in the running speed by changing only the frequency characteristic of the analog filter. Therefore, the characteristic of the digital filter is irrelevant to the running speed.

\section{Verification of the inertial mid-chord offset method by running tests}

To verify that track irregularities can be measured by the inertial mid-chord offset method, we performed running tests by using a current track inspection car (type MAYA34). We installed an accelerometer, integrating circuit and gyroscope on the track inspection car and measured the displacement between car body and rail from the conversion output of the car simultaneously by the normal mid-chord offset method and inertial mid-chord offset method.
Figure 5 (a) shows the track irregularities measured by the inertial mid-chord offset method, and Fig. 5 (b) the track irregularity waveforms measured simultaneously by the normal mid-chord offset method in this test. Although the measured waveforms of the irregularity of alignment contain some noise due to the limited precision of the measuring devices, the waveforms in Figs. 5 (a) and (b) approximately agree with each other. Therefore, it is proved by this test that the inertial mid-chord offset method is a promising low-cost track irregularity inspection method, as it can measure track irregularities at sufficiently high precision for practical purposes.

As an advantageous feature, this method correctly outputs mid-chord offset irregularities. This is important information for the control of track profile in curved sections which cannot normally be obtained by the conventional inertial method. Another feature of this method is that it does not require any changing on existing track maintenance process or maintenance target value for midchord offset irregularity waveforms, in introducing a track inspection system based on this method. In this regard as well, it is a promising track inspection method.

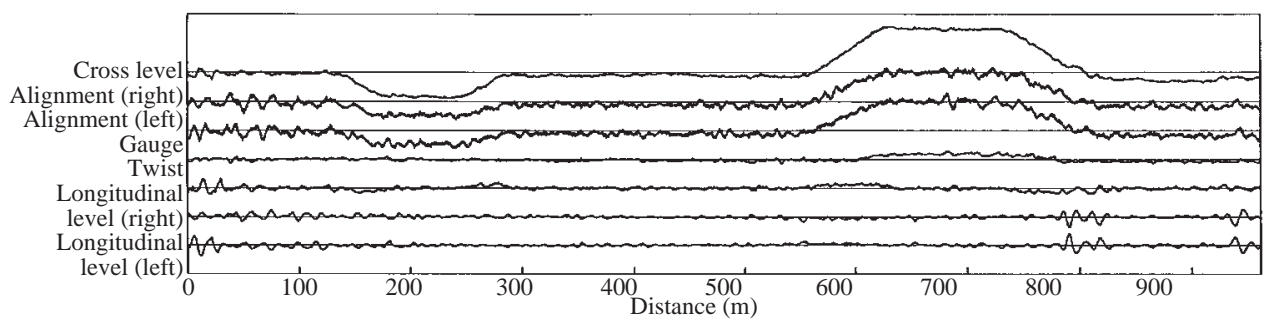

(a) Track irregularities measured by the inertial mid-chord offset method

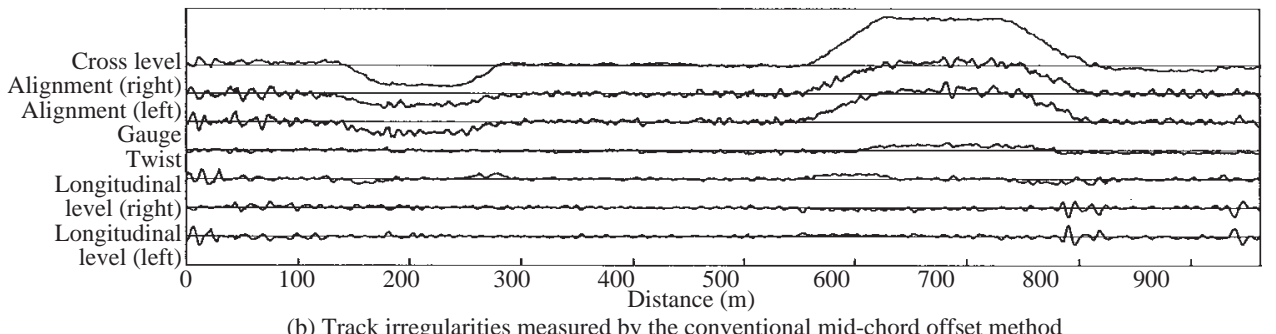

Fig. 5 Results of track inspection in running tests by the inertial mid-chord offset method 


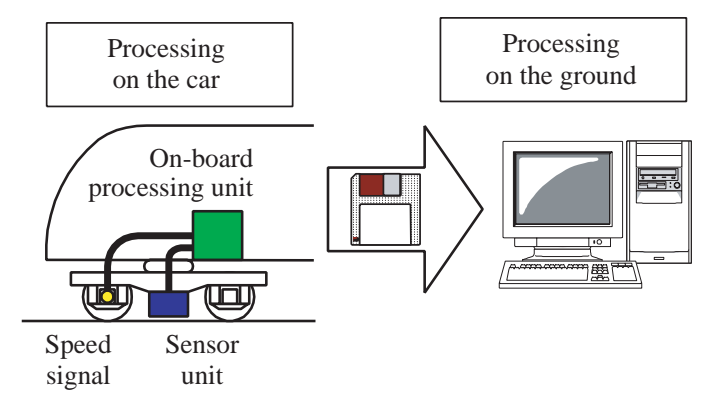

Fig. 6 Composition of the inertial mid-chord offset measuring device

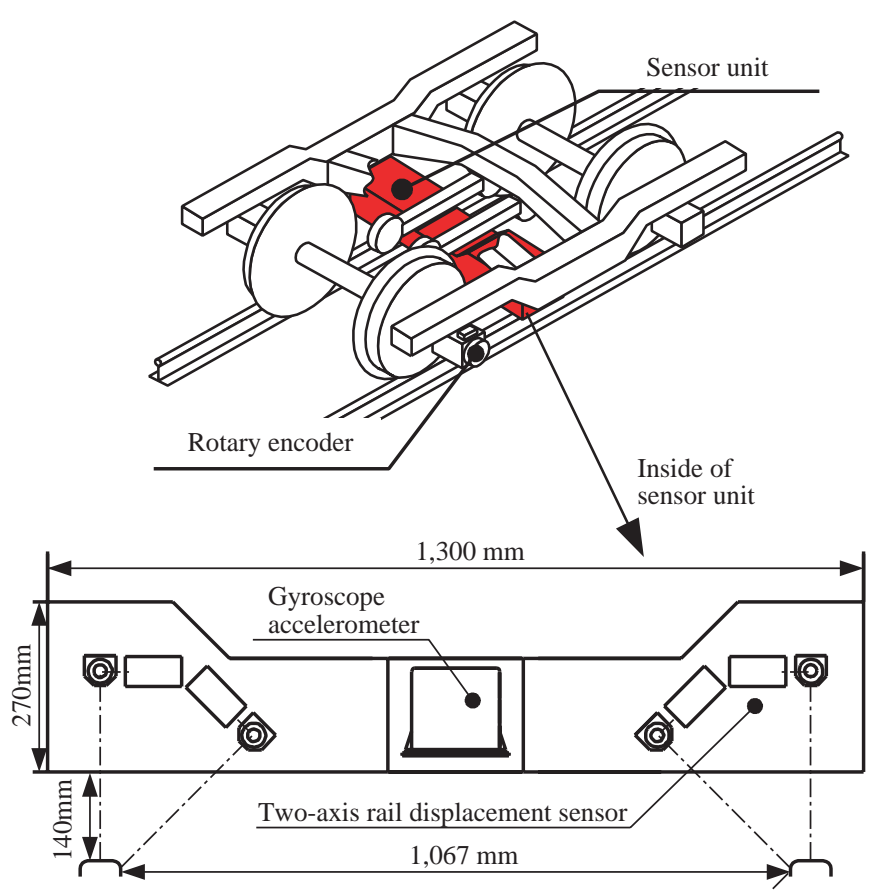

Fig. 7 Composition of the sensor unit

\section{Trial manufacture of track inspection device to use the inertial mid-chord offset method}

\subsection{Composition of the device}

Based on the test results, we manufactured a prototype track inspection device to use the inertial mid-chord offset method that can be mounted on a commercial railway vehicle. Figure 6 shows an image of the device. One of the most characteristic components is the sensor unit in Fig. 7 that will be fixed directly to the track frame. As heavy components are not installed on the axle box, this system does not adversely affect the car running performance and allows installation on commercial railway vehicles. This unit houses all sensors, which would otherwise be mounted on the car body and truck, to minimize the quantity of sensors and facilitate easier maintenance work. The gyroscope, which is installed near the accelerometer, is used to measure the irregularities of cross level and compensate for the inclination of accelerometer. In contrast to the inertial method whose precision is not high in the low-speed range, this method will ensure sufficiently

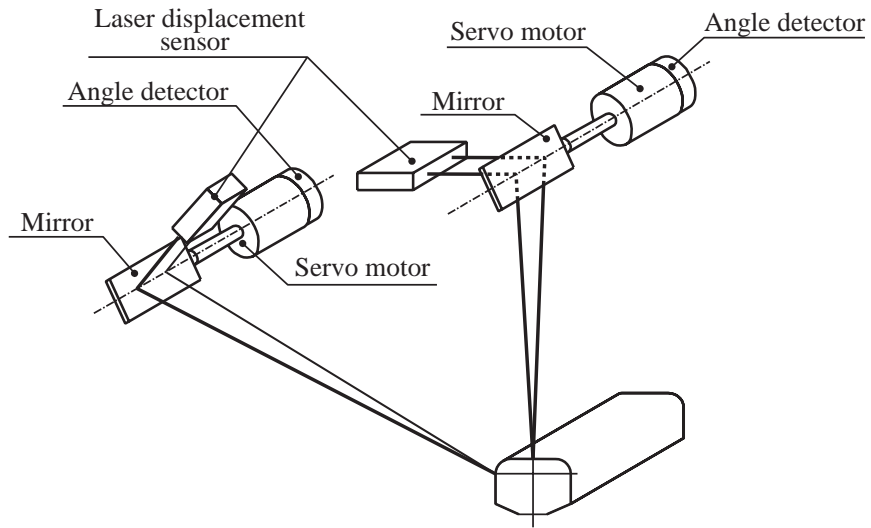

Fig. 8 Mechanism of the two-axis rail displacement sensor

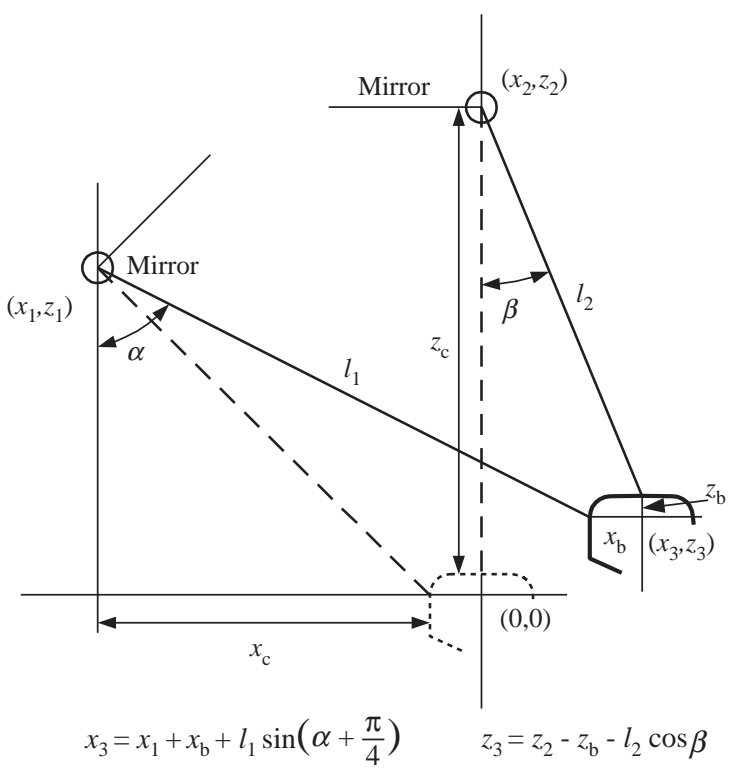

Fig. 9 Principle of displacement detection by the twoaxis rail displacement sensor

high precision for practical purposes down to the speed of about $15 \mathrm{~km} / \mathrm{h}$ due to the compensation of the accelerometer inclination.

\subsection{Development of two-axis rail displacement sensor}

One of the most characteristic components in the sensor unit is a newly developed optical two-axis rail displacement sensor, which is compact and lightweight, has a wide measuring range, and can be manufactured at a low cost. Figure 8 shows its mechanism. It uses a laser displacement sensor on the market to detect displacements by tracking the movement of rail head and side with a mirror driven by a servo motor. The output of the laser displacement sensor and the angle of mirror give the vertical and lateral rail displacements based on the principle of trigonometrical survey (see Fig. 9). If the relative positions of rail and laser displacement sensor change, the output of the displacement sensor changes to disrupt the triangle composed of the rail and mirrors. In this situation, the mirror angle changes to track the rail until the triangle closes. This control is also performed 


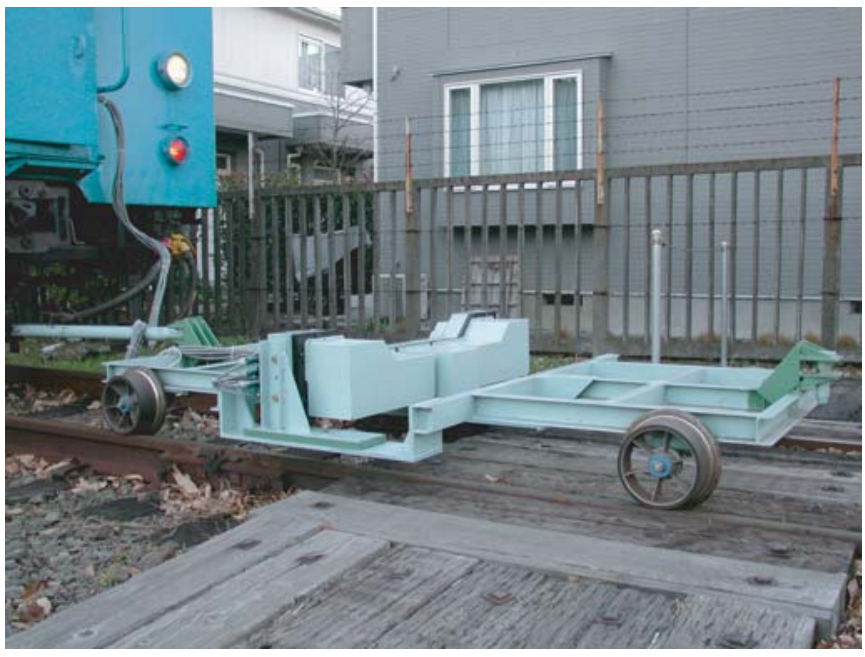

Fig. 10 Test to confirm the precision of the two-axis rail displacement sensor

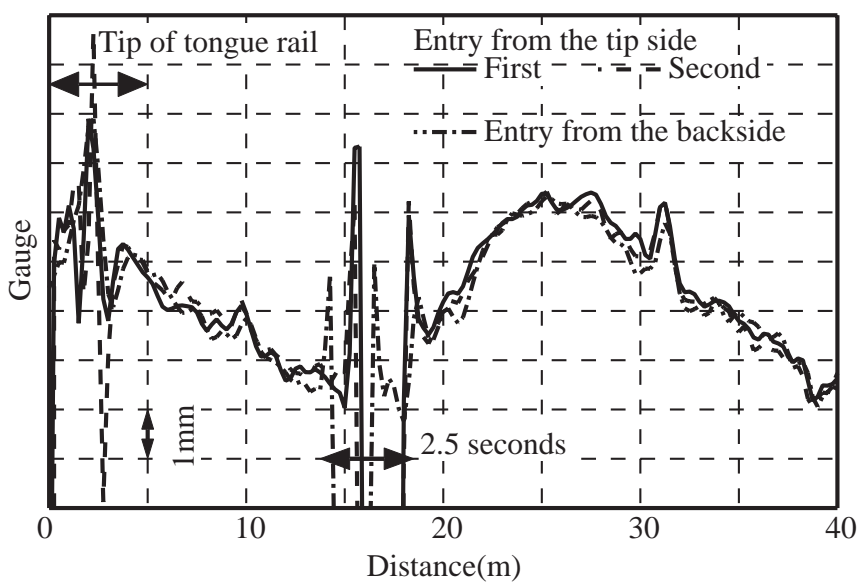

Fig. 11 Turnout passing tests of the two-axis rail displacement sensor by a board computer for factory automaton on the market to cut the cost of the system.

To confirm the precision of the two-axis rail displacement sensor, we measured track gauges at the test track of the Railway Technical Research Institute (See Fig. 10). Figure 11 shows the results of turnout passing tests. The maximum measurement error in these tests was $0.5 \mathrm{~mm}$ except in the tongue rail tip section.

This sensor has an algorithm to search a rail to be tracked that should be near the origin when the output of lateral displacement is $50 \mathrm{~mm}$ or over in passing the void section of turnout crossing. When it enters a turnout crossing from the nose tip side in particular, it tracks the wing rail for sometime before it catches the nose rail. Therefore, it takes a maximum duration of 2.5 seconds until the sensor correctly tracks the nose rail. When the track inspection device runs at high speed, it is possible that the sensor jumps at this transition section with the output interrupted momentarily. However, it is required to improve the precision of the sensor against this phenomenon in low speed operation. After improving the system for this reason, we will confirm the high speed response of the sensor.

\subsection{Data processing by the prototype device}

Figure 12 shows the flow of data processing by the prototype device. To minimize the volume of the vertical and lateral displacement data to be transferred from the on-board processing device to that on the ground, we transfer the intermediate data of the track center line irregularities. The intermediate data waveforms of track and cross level irregularities are the same as those obtained by the conventional track inspection cars except the scale factor. The effects of car movement are eliminated from the intermediate data by the calculation on the car. Therefore, identical waveforms are obtained from the same track section except errors.

The processing unit on the ground performs digital

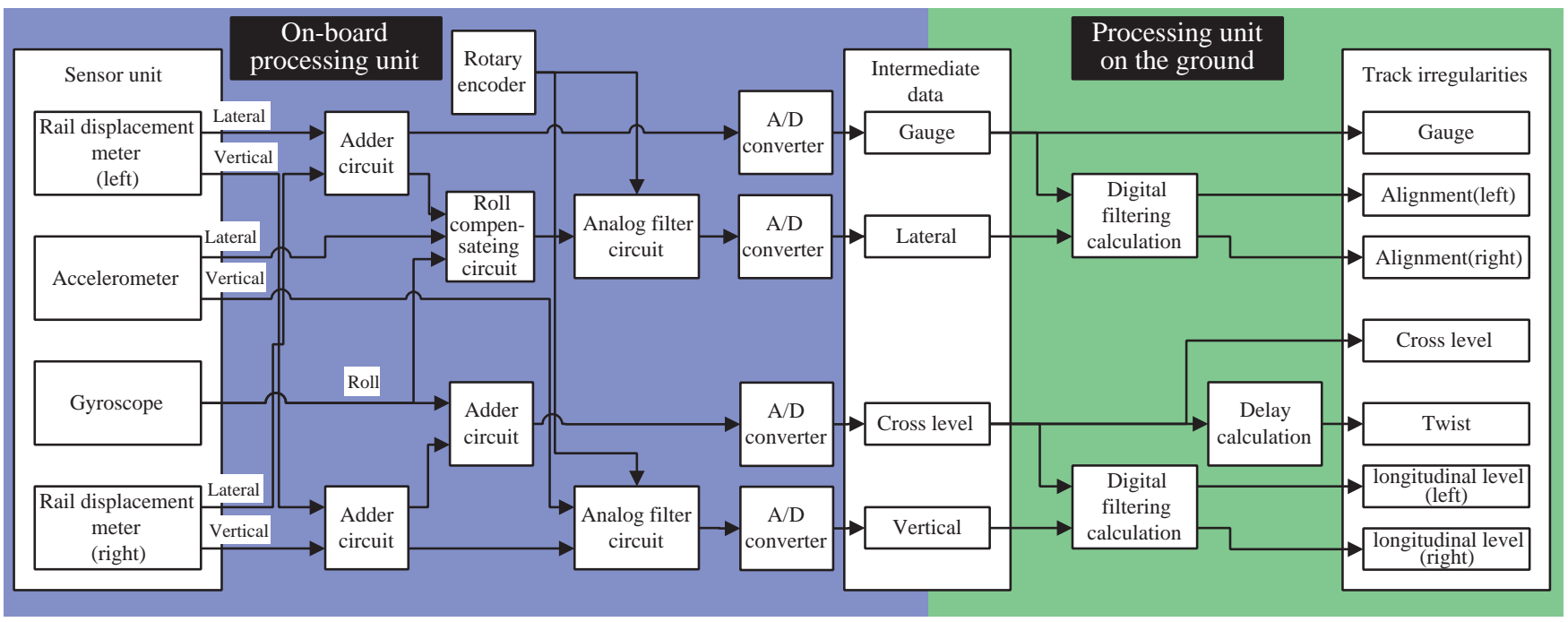

Fig. 12 Flow of the inertial mid-chord offset processing by the track inspection device 


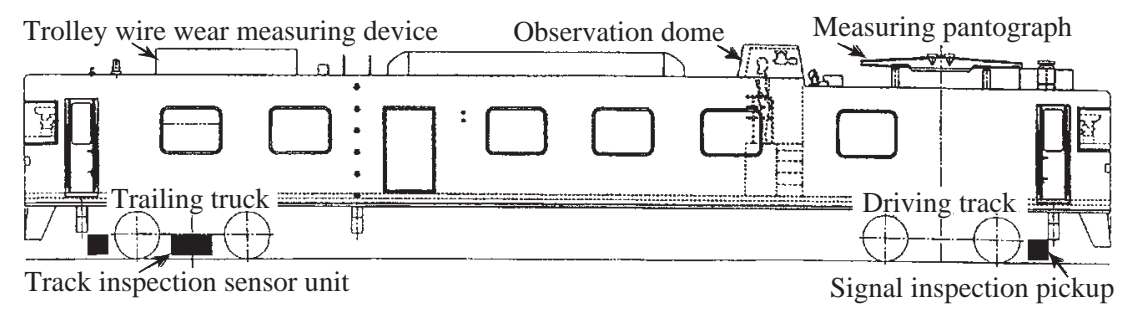

Fig. 13 Image of comprehensive DC inspection car

filtering to calculate the vertical and lateral displacements of both the left and right rails from the four items of intermediate data and also determines the twist. This processing uses personal computer software to cut the cost of the inspection system as a whole.

It is also possible to compose a track inspection car to perform all the processing shown in Fig. 12. Figure 13 is an image of a comprehensive inspection diesel car using this system to combine electrical and signal inspection systems.

\section{Conclusion}

The prototype inspection device to use the inertial mid-chord offset method is now under a running test. Through this test, we will confirm the precision of in- spection in the low- and high-speed ranges and clarify the performance when it is put in practical use.

A verification test was implemented with a current track inspection car in cooperation with the East Japan Railway Company. We want to express our sincere gratitude to those who kindly extended cooperation to this study.

\section{References}

1) Takeshita, K.:"A Study on the Inspection Method of Track Irregularities (in Japanese)," RTRI Report, Special No.25, pp.30-48, 1998.

2) Takeshita, K.:" Track Irregularity Inspection Method by Commercial Railway Vehicle," QR of RTRI, Vol.38, No.1, pp.6-12, 1997. 